\title{
Fotoexposição: Hábitos e Conhecimento de Estudantes de Medicina
} Sun Exposure: Habits and Knowledge of Undergraduate Medical Students

\author{
CAMILA ROOS MARIANO DA ROCHA \\ AMANDA TRONCO ${ }^{2}$ \\ FRANCESCO W. BORBA ${ }^{2}$ \\ JÚLIA LUNELLI ${ }^{2}$ \\ RAIANAMANFIO LAVRATTI ${ }^{2}$ \\ FABIANATONIAL ${ }^{1}$
}

\section{RESUMO}

Introdução: é conhecimento básico na formação médica que a radiação ultravioleta pode provocar diversos danos à pele e que a fotoproteção é importante para evitar tais prejuízos. Essa informação deve ser aprofundada e discutida durante a graduação em qualquer área das ciências da saúde. Ainda, acredita-se que o impacto da informação deve resultar na aplicação do conhecimento na rotina de quem o recebe. Por isso, o trabalho buscou identificar em uma determinada instituição de ensino se alunos de medicina adquirem e tornam prático esse ensinamento. Objetivo: avaliar o conhecimento sobre exposição solar de alunos de medicina correlacionando com a aplicabilidade dessa informação nos seus hábitos. Material e Métodos: foi realizado um estudo descritivo transversal com 207 estudantes do Curso de Medicina da Universidade de Passo Fundo - RS - de julho a dezembro de 2016. Os dados foram obtidos através de questionário padronizado autoaplicativo. Resultados: $93,6 \%$ dos alunos entrevistados responderam que já haviam recebido instruções sobre fotoproteção. $84,4 \%$ já haviam cursado a disciplina de dermatologia e, desses, $77,3 \%$ já haviam atendido pacientes com câncer de pele. Porém, $29,6 \%$ de todos os alunos entrevistados relataram utilizar protetor solar diariamente. Conclusão: os dados apontam que os estudantes avaliados sabem que a exposição solar inadequada tem por consequência efeitos nocivos sobre a pele, porém não apresentam o hábito de fotoproteção. 0 conhecimento está presente, porém não é aplicado.

\section{DESCRITORES}

Dermatologia .Fotoproteção.Radiação Solar.Ensino.

\begin{abstract}
Introduction: It is basic knowledge in medical training that ultraviolet radiation can cause extensive damage to the skin and that sun protection is important to avoid such damages. This information should be deepened and discussed among undergraduate students in any field of health sciences. In addition, it is believed that the impact of information should result in the application of knowledge in the routine of who receives it. Therefore, this work aimed to identify whether medical students of a given educational institution have acquired this information and put it into practice. Objective: To evaluate the knowledge of medical students about sun exposure correlating it with the applicability of this information in their routine. Material and Methods: This was a crosssectional, descriptive study was carried out with 207 students attending Medical School at the University of Passo Fundo RS - from July to December 2016. The data were obtained through a standardized self-applicable questionnaire. Results: $93.6 \%$ of students said they had received instruction on photoprotection; $84.4 \%$ had already attended the dermatology course, of which $77.3 \%$ had been in contact with skin cancer patients. However, only $29.6 \%$ of all students interviewed reported using sunscreen daily. Conclusion: The results showed that the surveyed students know that inadequate sun exposure has harmful effects on the skin, but do not have the habit of photoprotection. Knowledge is present but it is not applied.
\end{abstract}

\section{DESCRIPTORS}

Dermatology.Photoprotection.Solar Radiation.Education. 
$\mathrm{O}$ tipo de câncer mais comum é o câncer de pele, sendo que o número de casos da doença tem aumentado mundialmente. Os cancros da pele mais prevalentes são os do tipo não-melanoma. Eles são classificados em carcinoma basocelular e carcinoma de células escamosas ou carcinoma epidermoide. O carcinoma basocelular é o câncer de pele mais prevalente, porém o de menor agressividade $\mathrm{e}^{1,2}$. $\mathrm{O}$ melanoma é a neoplasia de pele responsável pela maioria dos óbitos decorrentes da doença, porém ele é menos frequente que os não-melanomas ${ }^{1,3}$.

O estimado aumento dos casos dessa doença pode ser controlado, já que medidas simples no estilo de vida, que devem ser estimuladas por profissionais da área da saúde, podem precaver essa patologia ${ }^{4}$. Um dos fatores de risco para o desenvolvimento do câncer de pele que pode ser manejado é a exposição inadequada aos raios ultravioleta. Evitar a exposição ao sol quando a incidência dos raios ultravioleta for elevada, utilizar filtro solar, chapéu e guarda-solsão maneiras efetivas de prevenção ao câncer de pele. Também é importante o incentivo à procura de um médico dermatologista na presença de qualquer alteração na pele para o tratamento precoce da doença e consequentemente redução de sua letalidade ${ }^{5}$.

Essas informações são básicas na formação de qualquer profissional da área de saúde e devem ser contempladas durante a graduação. Profissionais capacitados a transmitir esses ensinamentos e estimular a comunidade para a fotoproteção podem contribuir para uma possível redução na incidência de câncer de pele. Ainda, a aplicação do conhecimento na rotina desses alunos demonstra que a informação recebida obteve o merecido impacto, já que todos estão predispostos ao risco do desenvolvimento de câncer de pele. Com base nisso, foi realizada uma pesquisa com alunos de uma Faculdade de Medicina com o intuito de avaliar as informações transmitidas sobre câncer de pele e se elas geraram efeito nos hábitos desses estudantes.

\section{MATERIAL E MÉTODOS}

Foi realizado um estudo descritivo transversal com estudantes regularmente matriculados no curso de medicina da Universidade de Passo Fundo (UPF), Rio Grande do Sul. Os dados foram coletados no período de julho a dezembro de 2016. Para o cálculo do tamanho da amostra, tomou-se como ponto de partida o total de estudantes matriculados no primeiro semestre de 2016 (525 alunos), uma estimativa de prevalência de práticas de fotoproteção de $50 \%$ e nível de confiança de $93 \%$, resultando em uma amostra inicial mínima de 203 acadêmicos. A amostra obtida foi de 207 alunos, todos maiores de idade, em qualquer etapa do curso. O Curso de Medicina da Universidade de Passo Fundo possui no primeiro nível o Módulo de Saúde Coletiva que aborda a temática dos danos causados pela radiação solar sobre a pele e modos de prevenção, portanto mesmo os alunos que não haviam cursado a disciplina de dermatologia foram incluídos no estudo. Os dados foram coletados durante intervalos de aula, sendo incluídos estudantes voluntários que consentiram em participar da pesquisa após leitura do Termo de Consentimento Livre e Esclarecido. O estudo foi aprovado pelo Comitê de Ética em Pesquisa (CAAE: 57738216.3.0000.5342; número do parecer de aprovação: 1.710.395).

A abordagem dos alunos foi feita por meio de aplicação de questionário contendo perguntas abertas e fechadas. A avaliação do conhecimento dos alunos foi realizada com questionamentos mais amplos da temática, como: se já haviam cursado a disciplina de dermatologia, se receberam orientações sobre fotoproteção, se participaram de atendimento a paciente com câncer de pele, se realizam auto-exame, e, também, com perguntas mais específicas a respeito da identificação de lesões suspeitas, diferenciação de carcinoma basocelular de espinocelular, informações sobre melanoma (faixa etária mais acometida, apresentação, sítios de metástase). Para caracterizar a população de estudo foi considerado: período do curso de medicina, gênero, idade, histórico de câncer de pele na família, cor dos olhos, fototipo solar (I ao VI), sensibilidade e reação da pele quando exposta ao sol, sendo as últimas informações autorreferidas pelos alunos com base na classificação de Fitzpatrick ${ }^{6}$, definindo-se fototipo cutâneo tipo I a pele muito clara que queima com facilidade e nunca bronzeia; tipo II a pele clara que queima com facilidade e bronzeia pouco; tipo III a pele morena clara que queima e bronzeia moderadamente; tipo IV a pele morena moderada que queima pouco e bronzeia com facilidade; tipo $\checkmark$ a pele morena escura que queima raramente e bronzeia bastante; e tipo VI a pele negra que nunca queima e bronzeia sempre. Para a coleta de dados sobre o comportamento diante exposição solar os estudantes foram questionados sobre: frequência do uso de protetor solar; fator de proteção solar 
(FPS) utilizado (<15; 15; 30; 50; >50 FPS); hábito de reaplicar o protetor solar; uso de protetor solar durante atividades ao ar livre; hábito de proteção solar por meios físicos (roupas compridas, bonés/ chapéus, óculos de sol); uso de câmaras de bronzeamento; ocorrência de queimaduras solares; horários usuais de exposição solar; e momento habitual de exposição solar (atividade física; bronzeamento intencional; situações ocupacionais do cotidiano).

\section{RESULTADOS}

As características mais prevalentes da população em estudo foram: sexo feminino $(59,7 \%)$, com idade entre 21 e 25 anos (68\%), de olhos castanhos $(68,4 \%)$ e fototipo II $(46,1 \%)$. A distribuição geral do fototipo de todos os alunos avaliados está ilustrada na Figura 1 . 26,2\% dos entrevistados apresentaram histórico familiar de câncer de pele.

Em relação ao conhecimento adquirido no curso de medicina, $84,4 \%$ dos entrevistados já haviam cursado a matéria de Dermatologia, desses $77,3 \%$ já haviam atendido pacientes com câncer de pele, $74,2 \%$ relataram saber identificar lesões suspeitas e $70,4 \%$ disseram ter capacidade em diferenciar câncer de pele do tipo basocelular de espinocelular. Dentre os alunos que ainda não haviam cursado a disciplina de dermatologia 93,6\% relataram já ter recebido informação sobre fotoproteção, desses $15,4 \%$ assistiram a pacientes com câncer de pele e 34,6\% afirmaram saber identificar lesões suspeitas de câncer de pele.

Quando questionados sobre hábitos pessoais relativos à exposição solar 29,6\% afirmaram fazer uso diário de protetor solar, desses $39,8 \%$ afirmaram reaplicar o protetor solar uma vez e $22,9 \%$ mais de uma vez por dia. A frequência de uso de protetor solar dos estudantes entrevistados está ilustrada na Figura 2.

Dos usuários de protetor solar 58,3\% utilizavam fator de proteção 30 . Ao realizar atividades físicas com exposição solar a frequência no uso de protetor solar foi maior $(62,1 \%)$, porém $23,3 \%$ não utiliza outras formas de proteção. $46,8 \%$ dos estudantes entrevistados relataram já terem sofrido grandes queimaduras solares e $53,6 \%$ afirmaram que se expõem no sol entre as 10 e 16 horas. O uso de câmaras de bronzeamento foi referido por $4,4 \%$ dos alunos.

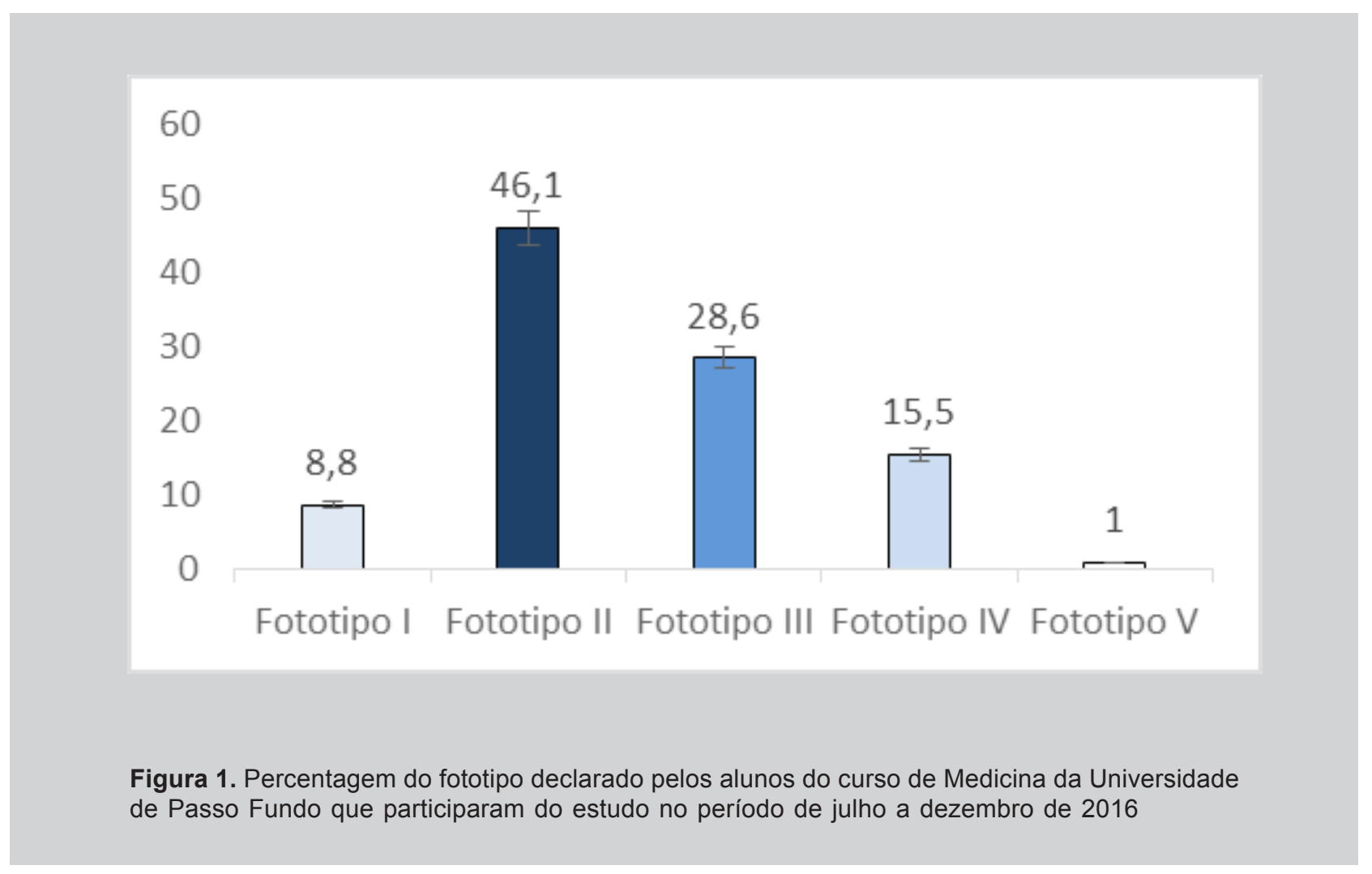




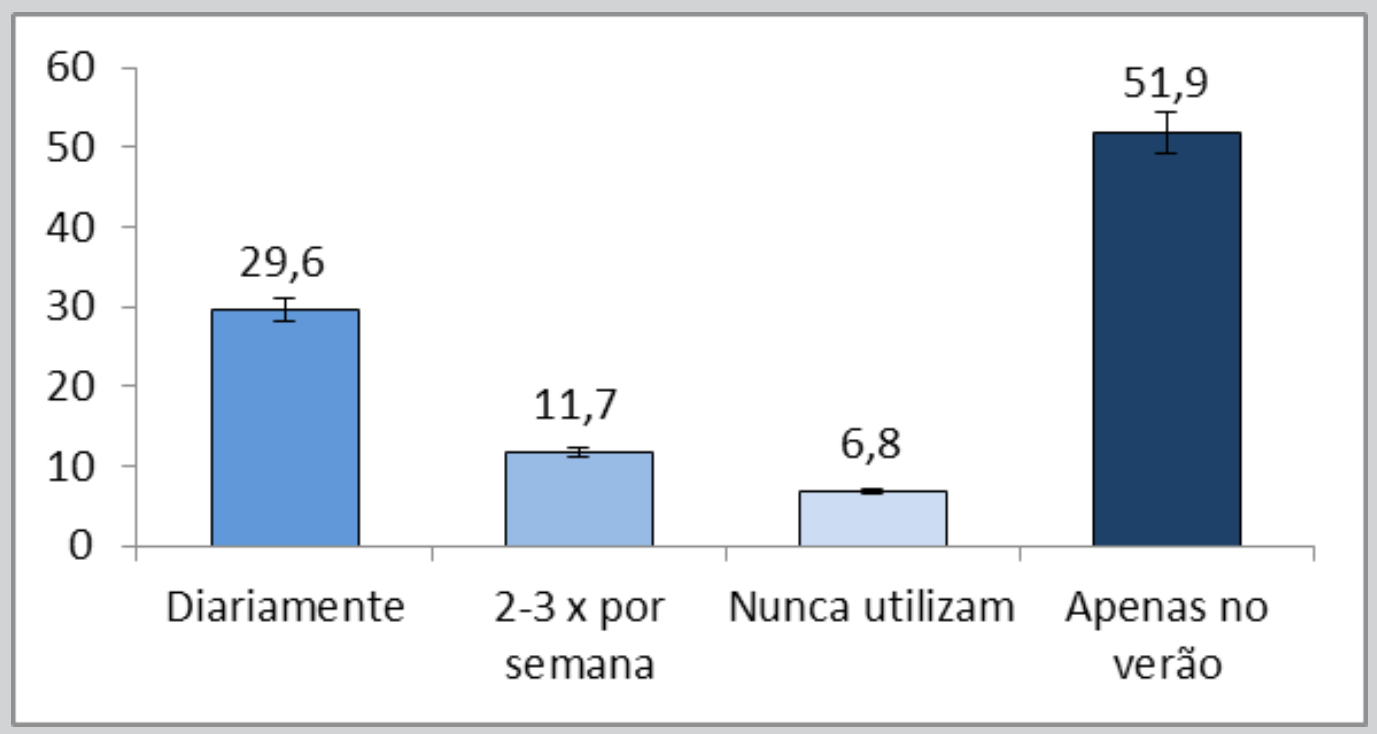

Figura 2. Percentagem de uso de protetor solar declarado pelos alunos do curso de Medicina da Universidade de Passo Fundo que participaram do estudo no período de julho a dezembro de 2016

\section{DISCUSSÃO}

Os resultados demonstram que $93.6 \%$ dos alunos entrevistados sabem que é necessário realizar a fotoproteção e como ela deve ser feita, incluíndo os alunos que não haviam cursado a disciplina de dermatologia, já que a abordagem desse tema também é realizada em momentos de formação inicial do curso, como no Módulo de Saúde Coletiva. No entanto, o conhecimento não gerou o impacto necessário e fica restrito à teoria, pois frente aos questionamentos dos hábitos diante à exposição solar $53,6 \%$ de todos os entrevistados relatam se expôr ao sol em horários inadequados e $58,7 \%$ não utilizam protetor solar ou fazem o uso apenas no verão. $O$ estudo revela que é necessário desenvolver uma metodologia de ensino em fotoproteção que além de transmitir a informação sensibilize o profissional. Pois, é fundamental a aplicação do conhecimento para uma sensibilização mais eficaz da comunidade na prevenção contra o câncer de pele.

A preocupação com os resultados expostos se agrava ao analisarmos que $54,9 \%$ da população estudada declara apresentar o fototipo I (pele muito clara que queima com facilidade e nunca bronzeia) ou fototipo II (pele clara que queima com facilidade e bronzeia pouco), portanto sofrem uma maior agressão frente aos efeitos nocivos da radiação solar.

Resultados semelhantes foram obtidos em uma Faculdade de Medicina de Curitiba, Paraná, onde a maioria dos entrevistados declarou possuir conhecimentos básicos sobre fotoproteção (65,3\%), porém apenas $36,5 \%$ usavam o filtro solar diariamente e $23,3 \%$ preferia se expôr ao sol em horários entre 10-16 horas. O histórico familiar de câncer de pele foi relatado por $24,6 \%$ dos estudantes. Além do estudo citado demonstrar que apesar dos conhecimentos adquiridos no curso de Medicina os alunos apresentavam hábitos inadequados de exposição solar, os autores relataram que as mulheres apresentaram melhor adesão às técnicas fotoprotetoras quando comparadas aos homens ${ }^{7}$.Outro estudo, aplicado em uma instituição de ensino privada de Taguatinga, 
Distrito Federal, nos cursos de Medicina, Educação Física, Direito e Comunicação Social relatou que $50 \%$ dos entrevistados preferiam tomar banho de sol entre 10 e 16 horas e menos de $25 \%$ fazia o uso diário de fotoprotetor ${ }^{8}$. Os trabalhos revelam que, apesar das informações recebidas durante a graduação,entre $25-36,5 \%$ dos estudantes de medicina faz uso de protetor solar diariamente e de 23,3-53,3\% afirmam se expor ao sol em horários inapropriados.

Ainda, outro trabalho realizado com médicos já graduados, não-oncologistas, revelou que quando questionados sobre as práticas preventivas sobre os cânceres mais comuns a maior parte das respostas constatadas não se adequou as recomendações de entidades como o Instituto Nacional do Câncer (INCA), a American Cancer Society (ACS) e a Canadian Task Force (CTF). Os médicos afirmaram que o fato ocorre pela falta de consensos de prevenção e de conhecimento em relação aos já existentes. As práticas médicas foram comparadas com as medidas preventivas apresentadas pelos consensos em câncer de mama, próstata, colo uterino, colorretal, pulmão e pele não-melanoma. Os autores revelam uma maior deficiência na aplicação dos consensos para a prevenção do câncer mama e colorretal. Para o câncer de pele não-melanoma foi obtido um índice de conformidade entre a prática médica e o consenso de $51,42 \%$. Fato que alerta para a necessidade de elaboração e padronização de consensos em práticas de prevenção de doenças

\section{REFERÊNCIAS}

1. Olinger RC. Hábitos de fotoproteção e fotoexposição entre estudantes de Medicina na Universidade Federal de Santa Catarina. Trabalho de Conclusão de Curso de Graduação em Medicina.Florianópolis, Universidade Federal de Santa Catarina; 2012. 34p.

2. Ministério da Saúde. Instituto Nacional do Câncer. ABC do Câncer: abordagens básicas para o controle do câncer. Rio de Janeiro:Inca; 2011. 128p. pelas entidades de classe ${ }^{9}$.

Os dados obtidos nesse trabalho associados com informações obtidas em outros estudos permitem concluir que tanto na nossa realidade como em outras localidades os estudantes da medicina não estão sensibilizados da importância da fotoproteção, pois não aplicam os conhecimentos teóricos na prática cotidiana. Mesmo sabendo dos riscos da radiação solar em horários inadequados e conhecendo formas de fotoproteção, estudantes e médicos mostraram resultados que sugerem ser necessária a retomada dessa questão, buscando uma abordagem didática que promova também a mudança no hábito de quem adquire o conhecimento. É possível que os resultados reflitam uma característica que pode ser atribuída à população em geral, na qual o impacto para a mudança de hábitos inadequados com o intuito de prevenir o desenvolvimento de determinada patologia, mesmo aquelas com os piores prognósticos, só ocorre quando a problemática torna-se uma realidade muito próxima do indivíduo, principalmente se observarmos que a taxa de histórico familiar de câncer de pele é de24,6 $-26,2 \%$ nos trabalhos apresentados.

\section{CONCLUSÃO}

Os resultados obtidos demonstraram que os estudantes detêm o conhecimento sobre fotoproteção. Entretanto, eles não aplicam os ensinamentos na sua rotina diária.
3. Paschoal F. Atualizações no estadiamento do melanoma. Publicação Oficial do Grupo Brasileiro de Melanoma. 2017; 19 (76):2.

4. INCA. Incidência de Câncer no Brasil: estimativas 2016.

5. INCA. Prevenção de Câncer de pele não melanoma.

6. Fitzpatrick $T$. The validity and practically of sun reactive skin types I throught VI. Arch. Dermatol. 1988; 124:869871. 
7. Purim KSM, Wroblevski FC. Exposição e proteção solar dos estudantes de Medicina de Curitiba (PR). Revista Brasileira de Educação Médica. 2014; 38(4):477-485.

8. Castilho IG, Sousa MAA, Leite RMS. Fotoexposição e fatores de risco para câncer de pele: uma avaliação de hábitos e conhecimentos entre estudantes universitários. Anais Brasileiros de Dermatologia. 2010; 85(2):173-178.

9. Tucunduva LTCM, de Sá VHLC, Koshimura ET, Prudente FVB, dos Santos AF, Samano EST, Costa LJM, Giglio AD. Estudo da atitude e do conhecimento dos médicos não oncologistas em relação às medidas de prevenção e rastreamento do câncer. Revista de Associação Médica Brasileira. 2004; 50(3):257-262.

\section{Correspondência}

Fabiana Tonial

Universidade de Passo Fundo, Instituto de Ciências Biológicas, BR 285, São José, Passo Fundo/RS, CEP 99052-900.

Email: fabianatonial@upf.br 